\title{
Synthesis of PolyHEMA Hydrogels for Using as Biomaterials. Bulk and Solution Radical-Initiated Polymerization Techniques
}

\author{
Juliana Matos Seidel and Sônia Maria Malmonge \\ Laboratório de Engenharia Biomecânica, Centro de Tecnologia, \\ Universidade Estadual de Campinas, 13083-970 Campinas - SP, Brazil
}

Received: February 7, 200; Revised: July 4, 2000

\begin{abstract}
Dense and porous polyHEMA hydrogels were synthesized by bulk and solution radical-initiated polymerization techniques. The morphologic analysis (SEM) of obtained hydrogels showed an amorphous dense structure for the samples obtained by bulk polymerization and a porous structure with non connected porous as a result of bulk polymerization in the presence of water soluble crystals (sucrose $/ \mathrm{NaCl}$ ). The use of solution polymerization technique using different amounts of water as diluent resulted in the formation of a sponge-like structure with connected microporous in case of using water amount equal or higher than $57.5 \%$. The saline solution uptake and dynamic mechanical behavior of dense hydrogels were influenced by the amount of crosslinking between the polymer chains.
\end{abstract}

Keywords: hydrogel, poly(2-hydroxyethyl methacrylate), biomaterial

\section{Introduction}

Hydrogel is a class of polymeric material which have the ability to hold substantial amount of water, showing soft and rubbery-like consistency and low interfacial tension ${ }^{1}$. The structural feature of these materials dominates its surface properties, permeselectivity and permeability that gives hydrogels their unique and interesting properties and similarity of their physical properties to those of living tissue $^{2,3}$. Hydrogels are materials that have a potential for use in biomedical applications since they show some advantages in relation to other polymeric biomaterials ${ }^{3-6}$. The nature of the hydrogel structure, due to its high water content, allows the extraction of the undesirable reaction by-products before implantation and the flow of body fluids between the tissue and implant in vivo. The possibility of fabrication in various geometric forms and the easy alteration of its physical form allow the adjustment of the physical properties according to a specific application. Generally the physical characteristics of hydrogels are determined by the synthesis methods and parameters.

Hydrogels obtained from 2-hydroxyl methacrylate (HEMA) are very commonly studied for use as biomaterial in different applications. This material was first studied by Wicherle ${ }^{4}$ that suggested its use as soft contact lenses, but today there are still some difficult for using them as implant devices because of the low mechanical strength showed by the polyHEMA hydrogels.

According to Chirilla et al. ${ }^{7}$, the bulk polymerization of HEMA result in a glassy and transparent material that is considered non porous. By the other side, the solution polymerization of this monomer allow the formation of porous structures which are influenced by the type and amount of diluent used.

Optically transparent hydrogels in the dense form are used in soft contact lenses while porous hydrogel can be used as a sponge biomaterial such as a synthetic graft for the repair of cartilaginous, osseous and other tissues ${ }^{8-11}$. To improve the graft fixation in the implant site is desirable the tissue growth to the interior of the porous hydrogel structure. Thus, the pores of the material need to be interconnected and larger enough to permit the cell invasion and vascularization of the neoformed tissue ${ }^{9,12}$.

The fundamental knowledge concerning the mechanis s o5 
synthesis of low prices, sterilizable hydrogels with different physical characteristics.

\section{Experimental}

Dense polyHEMA hydrogel samples were prepared by thermal polymerization $\left(75^{\circ} \mathrm{C} / 2 \mathrm{~h}\right.$ ) using 2-hydroxyethyl methacrylate monomer (Aldrich, WI, EUA), trimetilol propane trimethacrylate crosslinking agent (Retilox, SP, Brazil) and benzoil peroxide (Laporte Chemicals, SP, Brazil), as the polymerization initiator. Different crosslinking agent amount was used: 1.0, 3.0 and 5.0\% (w/w).

In order to obtain porous sponge hydrogel samples, two different approaches were used: bulk polymerization of the monomer and crosslinking agent in presence of water soluble crystals and solution polymerization technique using water as a diluent for the monomer and crosslinking agent. In the former, sucrose (Merck) and $\mathrm{NaCl}$ (Merck) crystals of different sizes (125-250) $\mu \mathrm{m}$ e (250-350) $\mu \mathrm{m}$ were added to the monomer and crosslinking agent mixture before polymerization.

In case of solution polymerization, distilled and deionized water was added to the monomer solution in concentrations from $20 \%$ to $60 \%(\mathrm{w} / \mathrm{w})$. The obtained samples were characterized by measuring the saline solution uptake ( $\mathrm{NaCl}$ 0.15 Mol/L); scanning electron microscopy (SEM) using a JXA840 JEOL equipment.

In case of dense samples, the mechanical behavior was verified by dynamic mechanical analysis using a DMA 242 NETZSCH equipment. For the dynamic mechanical analysis dried samples were submitted to a sinusoidal deformation under tension using a $240 \mu \mathrm{m}$ for displacement amplitude and $1.0 \mathrm{~Hz}$ frequency.

\section{Results and Discussion}

Dense hydrogels obtained showed the behavior of a rigid and fragile material when dried and an elastomeric consistence when swelled in water. The samples prepared by bulk polymerization showed an amorphous morphology without porous (Fig. 1). The morphology in this case was independent of the crosslinking agent content.

Generally dense hydrogels show an amorphous structure since it is very difficult the arrangement of the macromolecules and formation of crystallites is in the presence of crosslinks ${ }^{1}$ 


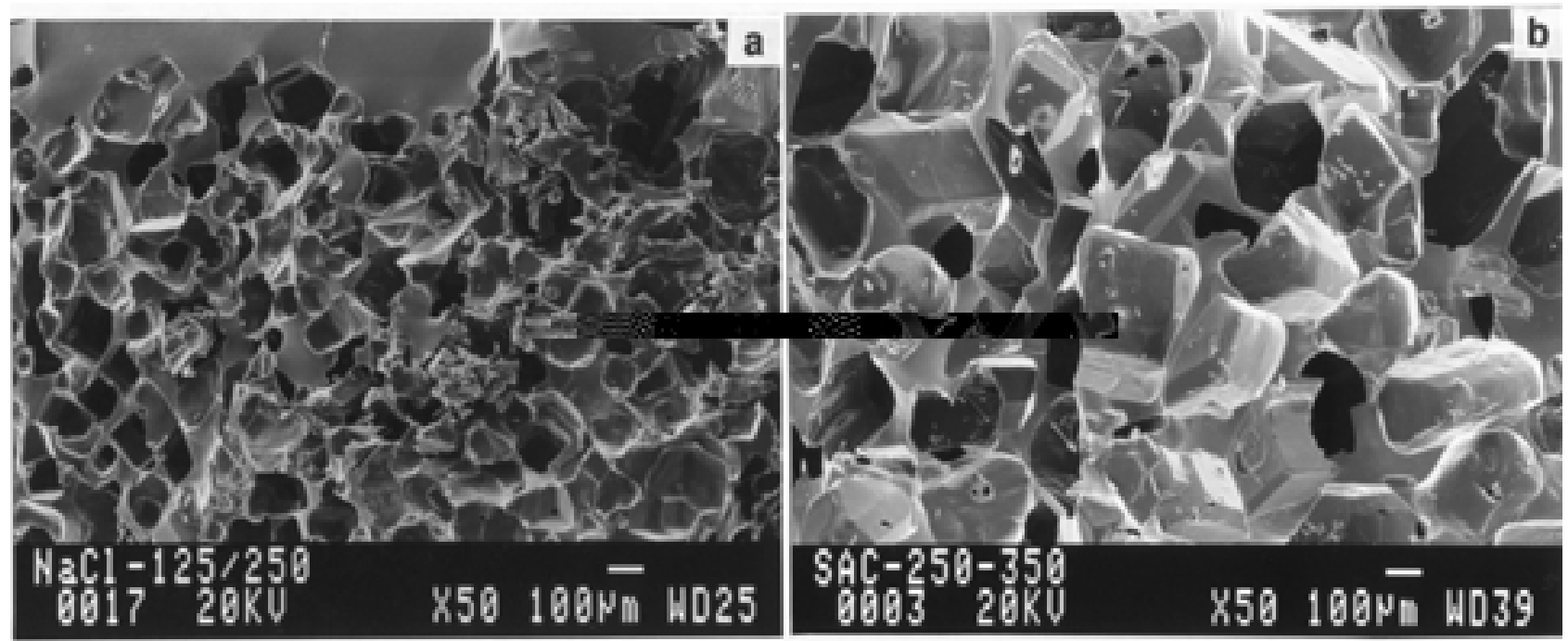

Figure 2. Photomicrography of polyHEMA porous sponge fractures in case of using: (a) $\mathrm{NaCl}$ crystals of diameter between $125-250 \mu \mathrm{m}$ and (b) sucrose crystals of diameter between $250-350 \mu \mathrm{m}$, in bulk polymerization.
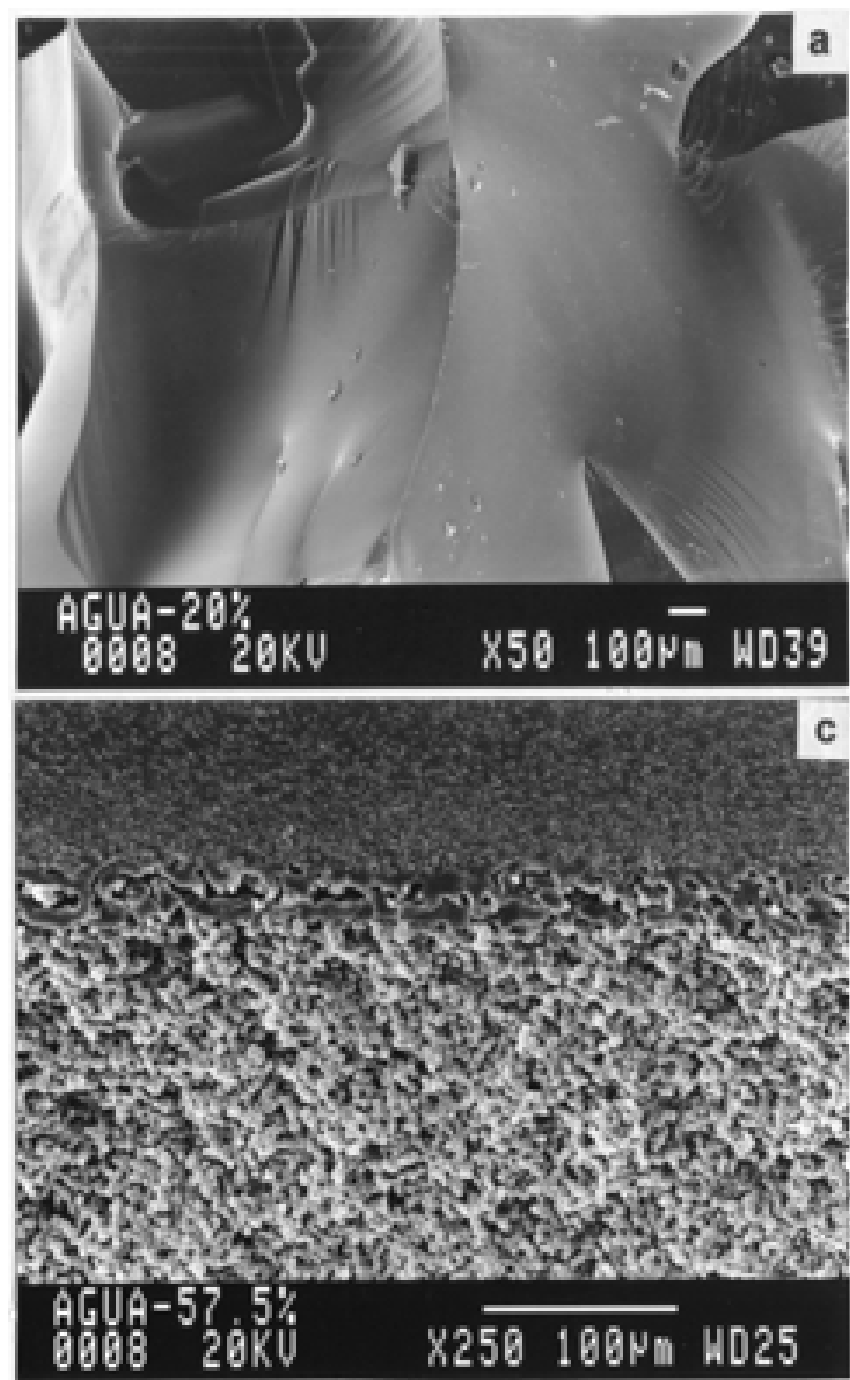
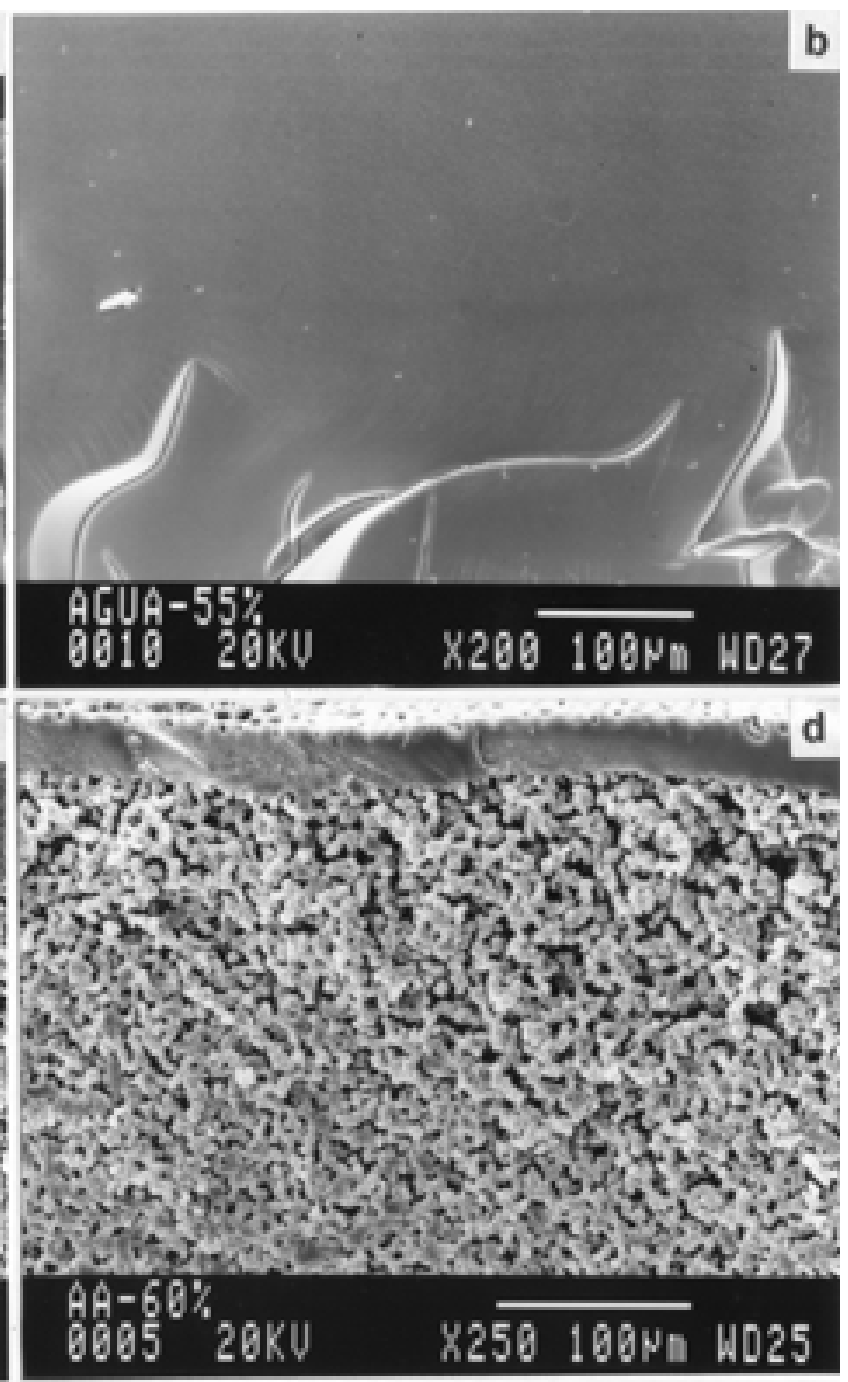

Figure 3. Photomicrography of polyHEMA porous sponge fractures in case of water concentration used in solution polymerization (a) $20 \%$; (b) $55 \%$; (c) $57.5 \%$ and (d) $60 \%$. 
crosslinking agents in the solution polymerization of HEMA using water as a diluent. These authors have concluded that the porous formation is dependent of the capacity of water sorption by the network formed, which change with different crosslinking type and density. However these authors did not observe the effect of the diluent in the mechanical behavior of the hydrogels obtained.

The results for saline solution uptake of different hydrogels obtained in this study are shown in the Fig. 4. It was observed a decrease in the saline uptake for hydrogels with higher percentage of crosslinking agent content.

The capacity of hydrogels to absorb high amount of water is an advantage of this material for use as implant in soft tissue replacement/restoration. In this work the hydrogels saline solution uptake was measured in order to know its behavior in an in vivo condition. Hydrogel saline solution uptake was decreased as a result of the crosslinking agent content increase. The increase of crosslinking between the macromolecules caused the decrease of network flexibility, making difficult the accommodation of water in the interior of the polymer structure.

In case of the hydrogels obtained by solution polymerization technique, the crosslinking agent content remained constant and the saline solution uptake was increased for samples with a microporous structure, that is, for amount of diluent used equal or higher than $55,7 \%$ (Fig. 5). This is a result of porous structure formed.

The elastomeric consistency showed by water swelled hydrogels is another advantage of this material for use as implant. Besides this, depending on the case, the implant material needs to be mechanically resistant. In this study the mechanical behavior of dense hydrogel samples was verified by performing dynamic mechanical analysis. For a material submitted to dynamic loading, the stress and strain responses are out of phase:

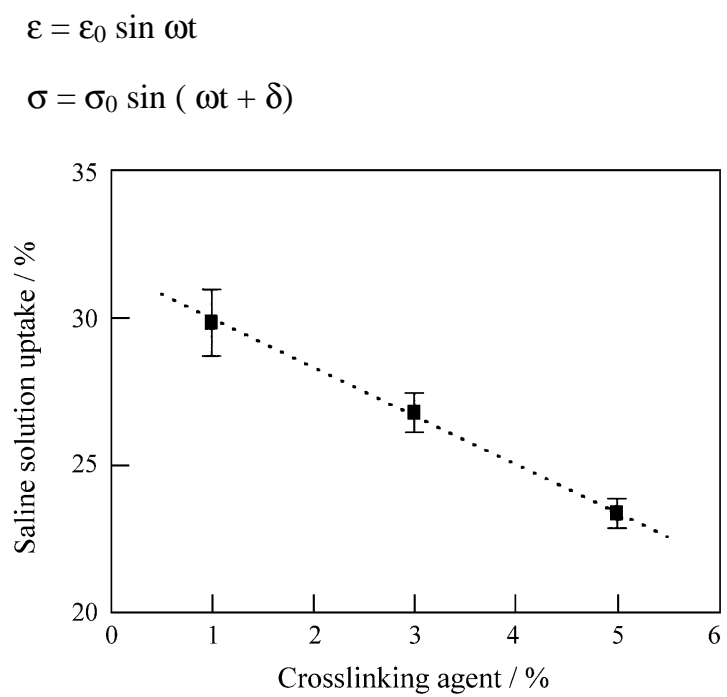

Figure 4. Saline solution uptake of polyHEMA with different crosslinking agent content.

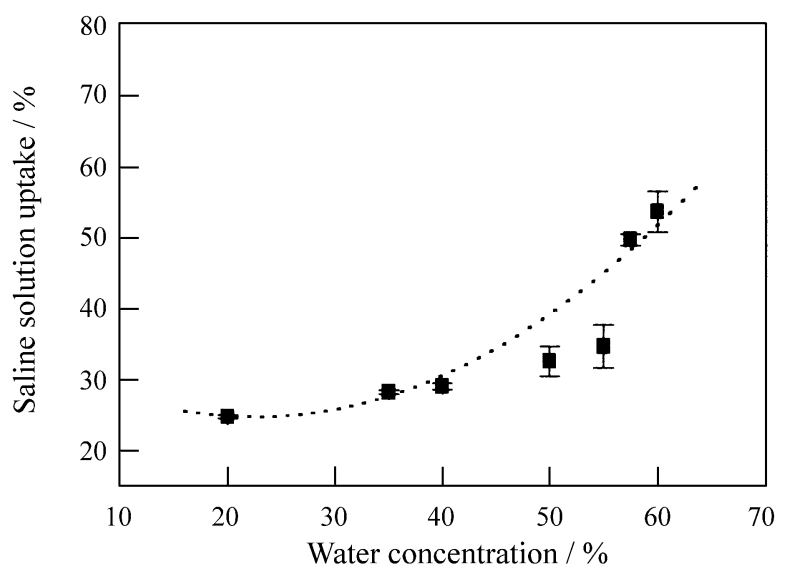

Figure 5. Saline solution uptake of polyHEMA obtained by solution polymerization using different amount of water as diluent.

where $\varepsilon$ is the strain, $\sigma$ is the stress, $\omega$ is the angular frequency $(2 \pi f)$ and $\delta$ is the phase angle by which stress leads to strain. The complex modulus $\left(\mathrm{E}^{*}\right)$, of a material under dynamic loading, is defined as the ratio between the stress amplitude and strain amplitude $\left(E^{*}=\sigma_{0} / \varepsilon_{0}\right)$. In this study, stiffness of the hydrogels under study was measured by the complex modulus.

The dynamic mechanical analysis showed changes on the storage modulus (E') and on glass transition temperature $(\mathrm{Tg})$ of bulk polymerized hydrogels with different crosslinking agent content (Table 1) and in case of solution polymerization for different amounts of diluent used (Table 2 ). The storage modulus ( $\left.E^{\prime}\right)$ is related to the rigidity of the material. Increasing on crosslinking agent content led to a decrease in the macromolecular network flexibility and consequent increase on the material rigidity. Besides this, a crosslinking agent content increase caused an increase in the glass transition temperature ( $\mathrm{Tg}$ ), since more energy is needed for material softening.

The polymerization of crosslinked polyHEMA in presence of water did not cause a significant change on the storage modulus (E'), but resulted in a decrease in the glass transition temperature (Tg). Probably this was a consequence of the polymer arrangement during polymerization. Water acts as a plasticiser, decreasing the interactions between polymer chains. If water is present during the polymer network synthesis, some water molecules remains

Table 1. Storage modulus (E') and glass transition temperature ( $\mathrm{Tg}$ ) of polyHEMA with different crosslinking agent content measured by dynamic mechanical analysis.

\begin{tabular}{lcc}
\hline Crosslinking agent content $(\%)$ & $\mathrm{E}^{\prime}(\mathrm{MPa})$ & $\operatorname{Tg}\left({ }^{\circ} \mathrm{C}\right)$ \\
\hline 1 & 3596 & 95.9 \\
2 & 3485 & 94.6 \\
3 & 4328 & 99.9 \\
\hline
\end{tabular}


Table 2. Storage modulus (E') and glass transition temperature (Tg) of polyHEMA with different amount of water used during the synthesis, measured by dynamic mechanical analysis.

\begin{tabular}{lcc}
\hline Amount of water $(\%)$ & $\mathrm{E}^{\prime}(\mathrm{MPa})$ & $\mathrm{Tg}\left({ }^{\circ} \mathrm{C}\right)$ \\
\hline 0 & 3485 & 94.6 \\
20 & 3740 & 84.5 \\
40 & 3568 & 86.0 \\
\hline
\end{tabular}

entrapped and strongly bonded to the polymer crosslinked structure $^{13}$ causing influences on the polymer Tg. The dynamic mechanical tests was carried out with dried samples of hydrogel in order to obtain information related to polymer network structure. The complete elimination of inside water for hydrogels is very difficult.

The mechanical strength was not evaluated, but it is known that it is decreased by the presence of pores in the hydrogel structure. Besides this, the results obtained in this study allow to conclude that the presence of water as a diluent for the monomer during the hydrogel synthesis do not change the rigidity of the polymer network at the environment temperature if the crosslinking agent amount do not change.

Results obtained in this work showed that the synthesis parameters for polyHEMA hydrogels determine its physical chemical characteristics. Thus the synthesis technique and parameters have to be chosen according to the desirable hydrogel characteristics.

\section{Conclusions}

The choice of synthesis technique for poly(2-hydroxyethyl methacrylate) hydrogels should be done according to the characteristics desirable for these materials since its physical, chemical and mechanical characteristics changes according to the synthesis method and parameters used. Bulk radical-initiated polymerization of poly(2-hydroxyethyl methacrylate) result in dense hydrogels with mechanical behavior influenced by the crosslinking density. It is possible to obtain poly(2-hydroxyethyl methacrylate) hydrogels with an interconnected porous structure by using the solution radical-initiated polymerization technique with water as a diluent.

Poly(2-hydroxyethyl methacrylate) hydrogels shown to be a class of materials that have great versatility, with a broad range of possible uses in the biomedical area since these materials can be easily prepared in different forms and are sterilizable in boiling water or in an autoclave.

\section{Acknowledgements}

The authors are grateful to CNPq (PIBIC), FINEP PRONEX and FAPESP (1996/10201-6) for their financial support.

\section{References}

1. Kudela, V. Hydrogels. In: Jacqueline IK, eds. Encyclopedia of Polymer Science and Engineering. Wiley Interscience, p. 783-807, 1976.

2. Hamilton, C.J.; Murphy, S.M.; Tighe, B.J. Polymer, v. 29, p. 1887-1893, 1988.

3. Ratner, B.D.; Hoffman, A. ACS Symp Ser, v. 31, p. 1-36, 1976.

4. Wichterle, O.; Lim, D. Nature , v. 185, p. 117-118, 1960.

5. Pedley, D.G.; Skelley, P.J.; Tighe, B.J. Brit Polymer J, v. 12, p. 99-110, 1980.

6. Ratner, B.D. Biomedical Applications of Hydrogels: Review and Critical Appraisal. In Williams DF, ed. Biocompatibility of Clinical Implant Materials. Flórida CRC, p. 145-175, 1981.

7. Chirila, T.V.; Constable, I.J.; Chen, Y.C.; Griffin, B.J. Polymer International, v. 32 n. 3, p. 221-232, 1993.

8. Chirila, T.V.; Constable, I.J.; Crawford, G.J.; Vijayasekaran, S.; Thompson, D.E.; Chen, Y.C.; Fletcher, W.A.; Griffin, B.J. Biomaterials, v. 14, n. 1, p. 26-38, 1993.

9. Oxley, H.R.; Corkhill, P.H.; Fitton, J.H.; Tighe, B.J. Biomaterials, v. 14, n. 14, p. 1064-1072, 1993.

10. Kon, M.; De Visser, A. Plast Reconstr Surg, v. 67, n. 5, p. 289-293, 1981.

11. Malmonge, S.M.; Zavaglia, C.A.C.; Belangero, W.D. 13th European Conference on Biomaterial, Gotborg, Sweden, p. 73, 1997.

12. Spector, M.; Miller, M.; Beals, N. Porous Materials. In: Webster, J.G., ed. Encyclopedia of Medical Devices and Instrumentation,_John Wiley \& Sons, 1988.

13. Davis, T.M.; Huglin, B.; Yip, D.C.F. Polymer, v. 29, p. 701-706, 1988. 